\title{
Paratingent Derivative Applied to the Measure of the Sensitivity in Multiobjective Differential Programming
}

\author{
F. García and M. A. Melguizo Padial \\ Departamento de Matemática Aplicada, Escuela Politècnica Superior, Universidad de Alicante, \\ San Vicente del Raspeig, 03080 Alicante, Spain
}

Correspondence should be addressed to M. A. Melguizo Padial; ma.mp@ua.es

Received 3 February 2013; Revised 25 March 2013; Accepted 25 March 2013

Academic Editor: Jen-Chih Yao

Copyright ( 2013 F. García and M. A. Melguizo Padial. This is an open access article distributed under the Creative Commons Attribution License, which permits unrestricted use, distribution, and reproduction in any medium, provided the original work is properly cited.

We analyse the sensitivity of differential programs of the form $\operatorname{Min} f(x)$ subject to $g(x)=b, x \in D$ where $f$ and $g$ are $\mathscr{C}^{1}$ maps whose respective images lie in ordered Banach spaces. Following previous works on multiobjective programming, the notion of $T$-optimal solution is used. The behaviour of some nonsingleton sets of $T$-optimal solutions according to changes of the parameter $b$ in the problem is analysed. The main result of the work states that the sensitivity of the program is measured by a Lagrange multiplier plus a projection of its derivative. This sensitivity is measured by means of the paratingent derivative.

\section{Introduction}

The subject of this work is sensitivity analysis in vector programming. The model problem considered throughout the paper is of the form

$$
\begin{array}{ll}
\text { Min } & f(x) \\
\text { subject to } & g(x)=b \\
& x \in D .
\end{array}
$$

Here, and throughout this work, $f: D \subset X \rightarrow Y$ and $g$ : $D \subset X \rightarrow Z$ denote two $\mathscr{C}^{1}$ maps, $X$ a Banach space and $Y$ and $Z$ two ordered Banach spaces. The sensitivity of the problem is analysed by studying the quantitative behaviour of a nonsingleton set of optimal points when the parameter $b \in Z$ varies.

As the maps $f$ and $g$ lie in Banach spaces, the obtained results provide a general framework in which a wide range of problems can be studied; see [1].

To perform our analysis we use the so-called T-optimal solution; that is, solutions of the program characterized to become a minimum when the objective function is composed with a positive topological homomorphism $T$. For a fixed $T$, we measure the perturbation experienced by the whole set of the $T$-optimal solutions (not necessarily a singleton) when the right-hand side vector $b$ varies. The study is carried out by means of set-valued derivatives.

Tangent cones are the cornerstone of the notion of derivative of a set-valued map. There are different notions of tangency, and each of them provides a different cone. Experience shows that there are, mainly, four useful kinds of cones: Bouligand's contingent, adjacent, Clarke's tangent (or circatangent), and Bouligand's paratingent (see $[2,3])$. All the four correspond to different regularity requirements and they carry in themselves a wide and particular information about the local behaviour of sets. Once a concept of tangent cone is chosen, we can associate with it a notion of the derivative of a set-valued map. Derivatives obtained with the former cones play an important role in several branches of mathematics, for example, nonsmooth analysis, control theory, viability theory, and so forth.

The notions of derivative of set-valued maps have been used in many recent papers in the context of stability and sensitivity analysis; see, for example, [4-17]. In this paper we follow this research line, analysing the sensetivity of the differential program (1) by means of the paratingent derivative. In previous papers, the study of sensitivity of this problem has been carried out by using adjacent, contingent, 
and circatangent derivatives (see $[18,19]$ ). Therefore, this study completes the sensitivity analysis of problem (1) with the four main derivatives mentioned above.

Our view is that, on the one hand, the main theorem of this work (Theorem A) measures the specific kind of sensibility provided by the paratingent cone. On the other hand, this result measures the sensitivity of the problem in cases in which previous results do not. Next, we explain the former claim. Clarke cone has the nice property to be always a closed convex cone. Then, Clarke derivative is a closed convex process, that is, the set-valued analogous of a continuous linear operator. The price of this property, however, is quite high since this tangent cone may often be too small or even reduced to the singleton $\{0\}$; see [2, Chapters 2 and 4]. In that case, the corresponding derivative does not provide any information about the sensitivity of the problem. Paratingent cone is a natural generalization of Clarke cone. Although its definition is less restrictive, it holds the property of stability of the tangency with respect to perturbations around the point where the cone is taken, in the same way that Clarke cone does. Paratingent cone is bigger than Clarke cone, then paratingent derivative can measure stability when Clarke derivative fails. Contingent and adjacent cones are intermediate cones between Clarke and paratingent ones, but they lack the above-mentioned tangency's stability property. Finally, as the paratingent cone is the biggest one of the four considered cones, it can provide information about the sensitivity when the others fail; see Example 2 of Section 2. Let us note that paratingent derivative is also useful in works on optimality conditions [20], differential inclusions [21-23], dynamical systems and ergodic theory [24], differentiable maps [25], and differentiation theory [26].

Before presenting the main results of the work, it is necessary to introduce some terminology and notation. Let us fix an order complete Banach lattice $W$ and a positive linear and continuous surjective map $T: Y \rightarrow W$ such that its kernel has a topological supplement. It is said that a feasible $x_{b} \in D$ is a local T-optimal solution of (1) when there exists a neighbourhood $U_{x_{b}} \subset D$ of $x_{b}$ such that $T f\left(x_{b}\right) \leq T f(x)$ for every feasible $x \in U_{x_{b}}$. It is clear that every local T-optimal solution of (1) is a local optimal solution of the program, that is, $f\left(x_{b}\right)-f(x) \notin Y_{+} \backslash\{0\}$ for every feasible $x \in U_{x_{b}}$. If $T$ is a topological isomorphism satisfying some weak requirements, then the set of the $T$-optimal solutions is dense in the efficient line (see [27]), and thus, it constitutes a very suitable set to describe the full efficient line. An element $x \in D$ is said to be regular with respect to the problem (1) if the Fréchet differential, $g^{\prime}(x, \cdot)$, of $g$ at $x$ is surjective. $\mathscr{B}(Z, Y)$ will denote the Banach space of the continuous linear maps from $Z$ on $Y$ with the usual norm and by $\pi$, the natural projection from $Y$ onto the kernel of $T$, Ker $T$. Let $x_{b} \in D$ be a regular local $T$-optimal solution of (1) is represented, a map $G \in \mathscr{B}(Z, Y)$ is said to be a T-Lagrange multiplier of (1) associated with $x_{b}$ if $T f^{\prime}\left(x_{b}, \cdot\right)=T G g^{\prime}\left(x_{b}, \cdot\right)$ and $\pi f\left(x_{b}\right)=\pi G(b)$. In [6], it is proved that for every regular local $T$-optimal solution $x_{b} \in D$ of (1), there exists a $T$-Lagrange multiplier $G_{x_{b}} \in \mathscr{B}(Y, Z)$ associated with it. Now we can define the following key setvalued maps of the work.
Definition 1. In the context of the precedent paragraph, it is defined that

(i) $T$-perturbation map of (1), $\Upsilon: V \subset Z \leadsto Y$, by

$$
\begin{array}{r}
\Upsilon(b):=\left\{f\left(x_{b}\right): x_{b}\right. \text { is a regular local } \\
T \text {-optimal solution of }(1)\} ;
\end{array}
$$

(ii) $T$-dual perturbation map of (1), $\Psi: V \subset Z \leadsto$ $\mathscr{B}(Z, Y)$, by

$$
\Psi(b):=\left\{G_{x_{b}}: \text { it is a } T\right. \text {-Lagrange multiplier of }
$$

(1) associated with $\left.x_{b}\right\}$.

Now we can state the main result of the work. In this, it is represented by $P^{L_{\Upsilon}} \Upsilon\left(\right.$ resp., $\left.P^{L_{\Psi}} \Psi\right)$, the paratingent derivative of $\Upsilon$ (resp., $\Psi$ ) relative to $L_{\Upsilon}$ (resp., $L_{\Psi}$ ).

Theorem A. Let one fixes $L_{\Upsilon} \subset \operatorname{Graph}(\Upsilon),\left(b_{*}, f\left(x_{b_{*}}\right)\right) \in L_{\Upsilon}$, and $L_{\Psi}=\left\{\left(b, G_{x_{b}}\right) \in \operatorname{Graph}(\Psi):\left(b, f\left(x_{b}\right)\right) \in L_{\Upsilon}\right.$ and $G_{x_{b}}$ is associated with $\left.x_{b}\right\}$. If $\Psi$ is is lower semicontinuous, paraderivable relative to $L_{\Psi}$ at $\left(b_{*}, G_{x_{b_{*}}}\right) \in L_{\Psi}$ and $T \Psi$ is continuously Fréchet differentiable at $b_{*}$, then $\Upsilon$ is lower semicontinuous, paraderivable relative to $L_{\Upsilon}$ at $\left(b_{*}, f\left(x_{b_{*}}\right)\right)$, and

$$
\begin{aligned}
P^{L_{\Upsilon}} \Upsilon & \left(b_{*}, f\left(x_{b_{*}}\right)\right)(z) \\
& =G_{x_{b_{*}}}(z)+\pi P^{L_{\Psi}} \Psi\left(b_{*}, G_{x_{b_{*}}}\right)(z)\left(b_{*}\right), \quad \forall z \in Z .
\end{aligned}
$$

If $L_{\Upsilon}=\operatorname{Graph}(\Upsilon)$, the derivatives of the statement of the former result are denoted by $P \Upsilon$ and $P \Psi$. In this case, we obtain a more particular but simpler version.

Corollary B. Let one supposes that $\Psi$ is lower semicontinuous, paraderivable relative to $\operatorname{Graph}(\Psi)$ at $\left(b_{*}, G_{x_{b_{*}}}\right) \in \operatorname{Graph}(\Psi)$, $T \Psi$ is continuously Fréchet differentiable at $b_{*}$, then $\Upsilon$ is lower semicontinuous, paraderivable relative to Graph( () at $\left(b_{*}, f\left(x_{b_{*}}\right)\right)$, and

$$
\begin{aligned}
& P \Upsilon\left(b_{*}, f\left(x_{b_{*}}\right)\right)(z) \\
& \quad=G_{x_{b_{*}}}(z)+\pi P \Psi\left(b_{*}, G_{x_{b_{*}}}\right)(z)\left(b_{*}\right), \quad \forall z \in Z .
\end{aligned}
$$

The proof of Theorem A is based on the fact that the setvalued map $\check{\Psi}$, defined by $\check{\Psi}(b):=\Psi(b)(b)$, is paraderivable when $T$-dual perturbation map $\Psi$ is and $T \Psi$ is Fréchet differentiable. This is stated in Theorem 12 of Section 4. Besides, on that result, the paratingent derivative of $\check{\Psi}$ is expressed in terms of the paratingent derivative of $\Psi$, formula (58). This formula also holds even when $\Psi$ is not paraderivable. The former fact and the proof of Theorem A allows us to claim that (4) also holds even when $\Psi$ is not paraderivable. As a consequence, (4) provides a way to measure sensitivity of problem (1) when the other derivatives fail.

The paper is organized as follows. In Section 2, the necessary mathematical background is reviewed; mainly, basic 
definitions and characterizations of cones and derivatives are provided in a precise way. Section 3 is a technical part devoted to establish some results which will be useful in Section 5. In particular, condition $\mathscr{T}$ is introduced and after that we prove Theorem 4 which allows us, via property $\mathscr{T}$, to transfer the condition of paraderivability from an arbitrary set-valued map $\Sigma: V \leadsto \mathscr{B}(Z, Y)$ to that one $\check{\Sigma}: V \backsim Y$ defined by $\check{\Sigma}(b)=\Sigma(b)(b)$. After that we obtain some more technical results. For example, in Lemma 6, it is proved when the paraderivability can be transferred from $\check{\Sigma}$ to $\pi \check{\Sigma}$, and Proposition 9, which is the paratingent version of the useful Proposition 5.1.2 of [2], provides the paratingent derivative of the sum of a set-valued map and a single-valued map. The objective of Section 4 is to prove the main result of the work about the sensitivity of the problem (1). After some technical considerations, Theorem 12 is stated and proved. In its statement it can be seen that it is possible to transfer the paraderivability property from the set-valued $\Psi$ to $\check{\Psi}$. After that, Theorem A is proved. At the end of Section 4 an example that illustrates the main theorem can be seen.

\section{Cones and Derivatives}

Before introducing the notions of cone and derivative, we will recall (and will go into details) some definitions which were scarcely given in the former section. Let us recall that $X$ represents a Banach space, $Y, Z$, and $W$ ordered Banach spaces such that $W$ is an order complete Banach lattice (i.e., every nonempty bounded from below subset has an infimum in $W$ ). Let $Y_{+}, Z_{+}$, and $W_{+}$denote the respective positive convex cones of $Y, Z$, and $W$, and suppose that $Y_{+}$and $Z_{+}$are closed. The dual space of a Banach space $Z$ will be denoted by $Z^{*}$. The map $T: Y \rightarrow W$ is fixed throughout the paper and it is a positive $\left(T\left(Y_{+} \backslash\{0\}\right) \subset W_{+} \backslash\{0\}\right)$ linear and continuous surjective map such that $\operatorname{Ker} T$ has a topological supplement denoted by $Y_{T}$. The symbol $\widehat{T}$ represents the restriction of $T$ to $Y_{T}$. It follows from the open mapping theorem (Theorem 2.11 in [28]) that the inverse operator $\widehat{T}^{-1}$ is continuous.

Now let us introduce the notions and characterizations of cones and derivatives that will be used throughout this work (see [2,29] for further details). Let $S$ be a normed space, $A \subset S$ a nonempty set, $\bar{A}$ its clausure in the norm topology, and $x \in$ $\bar{A}$. the natural distance map from the point $s \in S$ to the set $A$ will be denoted by $d(A, s)$ and the first infinite ordinal by $\omega$. The Bouligand contingent cone $T_{A}(x)$ to $A$ at $x$ is defined by

$$
T_{A}(x):=\left\{v \in S: \liminf _{h \rightarrow 0^{+}} \frac{d(A, x+h v)}{h}=0\right\} .
$$

Therefore, $v \in T_{A}(x)$ if and only if, there exist two sequences: $\left\{h_{n}\right\}_{n<\omega} \subset \mathbb{R}$ converging to $0^{+}\left(h_{n} \rightarrow 0^{+}\right.$for short $)$and $\left\{v_{n}\right\}_{n<\omega} \subset S$ converging to $v$ (S э $v_{n} \rightarrow v$ for short), such that $x+h_{n} v_{n} \in A$ for every $n<\omega$. Let $L \subset S$ a nonempty set and $x \in \bar{A} \cap \bar{L}$. The Bouligand paratingent cone $P_{A}^{L}(x)$ to $A$ relative to $L$ at $x$ is defined by

$$
P_{A}^{L}(x):=\left\{v \in S: \liminf _{h \rightarrow 0^{+}, L \ni \widehat{x} \rightarrow x} \frac{d(A, \widehat{x}+h v)}{h}=0\right\} .
$$

When $L=A$, we set $P_{A}(x):=P_{A}^{A}(x)$. Therefore, $v \in P_{A}^{L}(x)$ if and only if, there exist three sequences: $h_{n} \rightarrow 0^{+}, L \ni$ $x_{n} \rightarrow x$, and $S \ni v_{n} \rightarrow v$ such that $x_{n}+h_{n} v_{n} \in A$ for every $n<\omega$. Then $P_{A}^{L}(x) \supset T_{A}(x)$. Besides, when $L=\{x\}$ we have $T_{A}(x)=P_{A}^{L}(x)$. Hence, behaviour of paratingent cone respect to perturbations around the point $x$ is stabler than that of contingent cone.

In Section 1, we noted that paratingent cone is the biggest cone among all of the cones cited there. The following example shows a situation in which contingent cone vanishes, whereas paratingent cone does not. On that, we consider the Banach space of all bounded real sequences, $\ell_{\infty}=\left\{\left(x_{n}\right) \epsilon\right.$ $\left.\mathbb{R}^{\omega}: \sup _{n}\left|x_{n}\right|<\omega\right\}$, endowed with the supremum norm, $\|\mathbf{x}\|_{\infty}=\sup _{n}\left|x_{n}\right|$ for every $\mathbf{x}=\left(x_{n}\right) \in \ell_{\infty}$. In addition, we will denote by 0 the real number and by $\mathbf{0}_{\ell_{\infty}}$ the zero element of $\ell_{\infty}$.

Example 2. Define the set-valued map $\Sigma:\{0\} \cup\left\{2^{-i}: i<\omega\right\} \subset$ $\mathbb{R} \leadsto \ell_{\infty}$ by $\Sigma(0)=\left\{\mathbf{0}_{\ell_{\infty}}\right\}$ and, for every $i<\omega$,

$$
\begin{aligned}
\Sigma\left(2^{-i}\right):= & \left\{(-2^{-i-1}, \ldots, \underbrace{-2^{-i-1}}_{i \text { th coordinate }}, \mathbf{0}_{\ell_{\infty}}),\right. \\
& \left.(0, \ldots, 0, \underbrace{2^{-i}}_{i+1 \text {-th coordinate }}, 2^{-i}, \ldots)\right\} .
\end{aligned}
$$

Then

$$
T_{\operatorname{Graph}(\Sigma)}\left(0, \mathbf{0}_{\ell_{\infty}}\right)=\left\{\left(0, \mathbf{0}_{\ell_{\infty}}\right)\right\}
$$

but

$$
\left(-\frac{1}{2},\left(\frac{1}{2}, \frac{1}{2}, \ldots\right)\right) \in P_{\operatorname{Graph}(\Sigma)}\left(0, \mathbf{0}_{\ell_{\infty}}\right) .
$$

Finally, we now introduce the type of derivatives that we will handle. Let $S_{1}$ and $S_{2}$ be two normed spaces, $F: A \subset$ $S_{1} \leadsto S_{2}$ a set-valued map, and $x_{0} \in \operatorname{Dom}(F):=\{x \in A$ : $F(x) \neq \emptyset\}$. It is said that $F$ is lower semicontinuous at $x_{0}$ if for every $y \in F\left(x_{0}\right)$ and any sequence $\operatorname{Dom}(F) \ni x_{n} \rightarrow x_{*}$, there exists a sequence $Y \ni y_{n} \rightarrow y$ such that $y_{n} \in F\left(x_{n}\right)$ for every $n<\omega$. Let us fix $(x, y) \in \operatorname{Graph}(F):=\{(x, y) \in$ $\left.S_{1} \times S_{2}: y \in F(x)\right\}$, the contingent derivative of $F$ at $(x, y) \in \operatorname{Graph}(F)$ is the set-valued map $D F(x, y): S_{1} \leadsto S_{2}$ defined by $\operatorname{Graph}(D F(x, y)):=T_{\operatorname{Graph}(F)}(x, y)$. Now let us fix also a subset $L \subset \operatorname{Graph}(F)$, the paratingent derivative of $F$ relative to $L$ at $(x, y) \in L$ is the set-valued map $P^{L} F(x, y)$ : $S_{1} \leadsto S_{2}$ defined by $\operatorname{Graph}\left(P^{L} F(x, y)\right):=P_{\mathrm{Graph}(F)}^{L}(x, y)$. If $L=\operatorname{Graph}(F)$, then $P^{L} F(x, y)$ is written as $P F(x, y)$. Finally, $F$ is said to be paraderivable relative to $L \subset \operatorname{Graph}(F)$ at $(x, y) \in L$ if $D F(x, y)=P^{L} F(x, y)$. As can be seen in [11], if $F$ is single-valued and Fréchet differentiable at $x$, then $F$ is derivable at $(x, F(x))$ and $D F(x, F(x))(u)=F^{\prime}(x, u)$ for every $u \in S_{1}$. However, in our framework, a single-valued map $F$ has to be continuously Fréchet differentiable at $x$ in order to be paraderivable relative to $L \subset \operatorname{Graph}(F)$ at $(x, F(x)) \in L$; in this case we have $P^{L} F(x, F(x))(u)=F^{\prime}(x, u)$ for every $u \in S_{1}$. 


\section{Regularity Condition and First Results on Paraderivability}

In this section the regularity condition $\mathscr{T}$ will be established. It will allow us to transfer the condition of paraderivability from a set-valued map $\Sigma: V \leadsto \mathscr{B}(Z, Y)$ to the correspond$\operatorname{ing} \check{\Sigma}: V \leadsto Y$ defined by $\check{\Sigma}(b):=\Sigma(b)(b)$. It is known that if $\Sigma$ is a single-valued and Fréchet differentiable map then $\check{\Sigma}$ is also Fréchet differentiable (see Lemma 11 in [4]).

From now on, the following notation will be used. Given a set-valued map $\Sigma: V \rightsquigarrow \mathscr{B}(Z, Y)$, the set-valued map $\check{\Sigma}$ : $V \leadsto Y$ will be defined by $\check{\Sigma}(b)=\Sigma(b)(b)$ for every $b \in V$. Given a set $L \subset \operatorname{Graph}(\Sigma)$, the set $\check{L}$ will be defined by $\check{L}=$ $\{(b, G(b)):(b, G) \in L\} \subset \operatorname{Graph}(\check{\Sigma})$.

Definition 3. A set-valued map $\Sigma: V \leadsto \mathscr{B}(Z, Y)$ is said to have property $\mathscr{T}(L)$ at $\left(b_{*}, G_{*}\right) \in L \subset \operatorname{Graph}(\Sigma)$ if after fixing the following four sequences:

(i) $h_{n} \rightarrow 0^{+}$;

(ii) $Z \ni b_{n} \rightarrow b \in Z$;

(iii) $\check{L} \ni\left(\widehat{b}_{n}, \widehat{G}_{n}\left(\widehat{b}_{n}\right)\right) \rightarrow\left(b_{*}, G_{*}\left(b_{*}\right)\right)$;

(iv) $\left\{G_{n}\right\}_{n<\omega} \subset \mathscr{B}(Z, Y)$ such that each $G_{n} \in \sum\left(\widehat{b}_{n}+h_{n} b_{n}\right)$ and there exists

$$
\lim _{n \rightarrow \omega} \frac{G_{n}\left(\widehat{b}_{n}+h_{n} b_{n}\right)-\widehat{G}_{n}\left(\widehat{b}_{n}\right)}{h_{n}} \in Y ;
$$

there exist three sequences

(1) $Z \ni \bar{b}_{n} \rightarrow b \in Z$;

(2) $\mathscr{B}(Z, Y) \ni \widetilde{G}_{n} \rightarrow G_{*}$ such that each $\widetilde{G}_{n} \in \sum\left(\widehat{b}_{n}\right)$ and $\widetilde{G}_{n}\left(\widehat{b}_{n}\right)=\widehat{G}_{n}\left(\widehat{b}_{n}\right)$;

(3) $\left\{\bar{G}_{n}\right\}_{n<\omega} \subset \mathscr{B}(Z, Y)$ verifying that each $\bar{G}_{n} \in \Sigma\left(\widehat{b}_{n}+\right.$ $\left.h_{n} \bar{b}_{n}\right)$, in such a way that

$$
\begin{aligned}
& \lim _{n \rightarrow \omega} \frac{\bar{G}_{n}\left(\widehat{b}_{n}+h_{n} \bar{b}_{n}\right)-\widetilde{G}_{n}\left(\widehat{b}_{n}\right)}{h_{n}} \\
& \quad=\lim _{n \rightarrow \omega} \frac{G_{n}\left(\widehat{b}_{n}+h_{n} b_{n}\right)-\widehat{G}_{n}\left(\widehat{b}_{n}\right)}{h_{n}} \in Y,
\end{aligned}
$$

such that there exits

$$
\lim _{n \rightarrow \omega} \frac{\bar{G}_{n}-\widetilde{G}_{n}}{h_{n}} \in \mathscr{B}(Z, Y) .
$$

The interpretation of the former definition can be as follows. It is not restrictive to suppose that the two sequences of linear and continuous maps, $\left\{G_{n}\right\}_{n}$ and $\left\{\widehat{G}_{n}\right\}_{n}$, related by the pointwise limit (11) are, in fact, related by the uniform version of this limit. In the next result we will see how, by means of property $\mathscr{T}$, it is possible to transfer the paraderivability from the set-valued map $\Sigma$ to the set-valued map $\check{\Sigma}$ defined above.
Theorem 4. Let $\Sigma: V \leadsto \mathscr{B}(Z, Y)$ be a set-valued map with property $\mathscr{T}(L)$ at $\left(b_{*}, G_{*}\right) \in L \subset G r a p h(\Sigma)$ and paraderivable relative to $L$ at $\left(b_{*}, G_{*}\right)$. Then $\check{\Sigma}$ is also paraderivable relative to $\check{L}$ at $\left(b_{*}, G_{*}\left(b_{*}\right)\right) \in \check{L}$ and

$$
\begin{aligned}
P^{\check{L}} \check{\Sigma}\left(b_{*}, G_{*}\left(b_{*}\right)\right)(z) \\
\quad=P^{L} \Sigma\left(b_{*}, G_{*}\right)(z)\left(b_{*}\right)+G_{*}(z), \quad \forall z \in Z .
\end{aligned}
$$

Proof. The paraderivability of $\check{\Sigma}$ is a direct consequence of the equality (14). Thus, let us begin the proof by showing the equality. the inclusion $C$ will be first proved. For this purpose

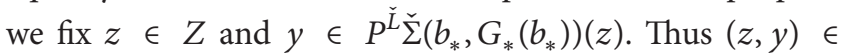
$P_{\operatorname{Graph}(\check{\Sigma})}^{\check{L}}\left(b_{*}, G_{*}\left(b_{*}\right)\right)$ and there exist $h_{n} \rightarrow 0^{+}, Z \ni b_{n} \rightarrow z$, $\check{L} \ni\left(\widehat{b}_{n}, \widehat{G}_{n}\left(\widehat{b}_{n}\right)\right) \rightarrow\left(b_{*}, G_{*}\left(b_{*}\right)\right)$, and $\left\{G_{n}\right\}_{n<\omega} \subset \mathscr{B}(Z, Y)$ such that each $G_{n} \in \Sigma\left(\widehat{b}_{n}+h_{n} b_{n}\right)$ and

$$
\lim _{n \rightarrow \omega} \frac{G_{n}\left(\widehat{b}_{n}+h_{n} b_{n}\right)-\widehat{G}_{n}\left(\widehat{b}_{n}\right)}{h_{n}}=y .
$$

Since $\Sigma$ verifies property $\mathscr{T}(L)$ at $\left(b_{*}, G_{*}\right)$, we can fix the three sequences of the second part of Definition 3. Let us recall that the following equality holds

$$
\begin{aligned}
\lim _{n \rightarrow \omega} & \frac{\bar{G}_{n}\left(\widehat{b}_{n}+h_{n} \bar{b}_{n}\right)-\widetilde{G}_{n}\left(\widehat{b}_{n}\right)}{h_{n}} \\
& =\lim _{n \rightarrow \omega} \frac{G_{n}\left(b_{n}+h_{n} b_{n}\right)-\widehat{G}_{n}\left(\widehat{b}_{n}\right)}{h_{n}} .
\end{aligned}
$$

We define

$$
G:=\lim _{n \rightarrow \omega} \frac{\bar{G}_{n}-\widetilde{G}_{n}}{h_{n}} \in \mathscr{B}(Z, Y) .
$$

Hence, $G \in P^{L} \Sigma\left(b_{*}, G_{*}\right)(z)$ because $L \ni\left(\widehat{b}_{n}, \widetilde{G}_{n}\right) \rightarrow\left(b_{*}, G_{*}\right)$, $h_{n} \rightarrow 0^{+}$,

$$
\begin{gathered}
\left(\bar{b}_{n}, \frac{\bar{G}_{n}-\widetilde{G}_{n}}{h_{n}}\right) \longrightarrow(z, G), \\
\left(\widehat{b}_{n}, \widetilde{G}_{n}\right)+h_{n}\left(\bar{b}_{n}, \frac{\bar{G}_{n}-\widetilde{G}_{n}}{h_{n}}\right) \\
=\left(\widehat{b}_{n}+h_{n} \bar{b}_{n}, \bar{G}_{n}\right) \in \operatorname{Graph}(\Sigma), \quad \forall n<\omega .
\end{gathered}
$$

From (15) and (16) we get

$$
\begin{aligned}
y & =\lim _{n \rightarrow \omega} \frac{G_{n}\left(\widehat{b}_{n}+h_{n} b_{n}\right)-\widehat{G}_{n}\left(\widehat{b}_{n}\right)}{h_{n}} \\
& =\lim _{n \rightarrow \omega} \frac{\bar{G}_{n}\left(\widehat{b}_{n}+h_{n} \bar{b}_{n}\right)-\widetilde{G}_{n}\left(\widehat{b}_{n}\right)}{h_{n}} \\
& =\lim _{n \rightarrow \omega}\left[\frac{\bar{G}_{n}-\widetilde{G}_{n}}{h_{n}}\left(\widehat{b}_{n}\right)+\bar{G}_{n}\left(\bar{b}_{n}\right)\right] \\
& =G\left(b_{*}\right)+G_{*}(z) .
\end{aligned}
$$


Finally, since $G \in P^{L} \Sigma\left(b_{*}, G_{*}\right)(z)$, then $y \in P^{L} \Sigma\left(b_{*}, G_{*}\right)$ $(z)\left(b_{*}\right)+G_{*}(z)$.

For the reverse inclusion, $\supset$, we fix $z \in Z$ and $y \in$ $P^{L} \Sigma\left(b_{*}, G_{*}\right)(z)\left(b_{*}\right)+G_{*}(z)$. Let us fix now $G \in P^{L} \sum\left(b_{*}, G_{*}\right)(z)$ such that $y=G\left(b_{*}\right)+G_{*}(z)$. By definition, there exist $L \ni$ $\left(\widehat{b}_{n}, \widehat{G}_{n}\right) \rightarrow\left(b_{*}, G_{*}\right), h_{n} \rightarrow 0^{+}, Z \times \mathscr{B}(Z, Y) \ni\left(b_{n}, G_{n}\right) \rightarrow$ $(z, G)$, such that each

$$
\begin{aligned}
& \left(\widehat{b}_{n}, \widehat{G}_{n}\right)+h_{n}\left(b_{n}, G_{n}\right) \\
& \quad=\left(\widehat{b}_{n}+h_{n} b_{n}, \widehat{G}_{n}+h_{n} G_{n}\right) \in \operatorname{Graph}(\Sigma) .
\end{aligned}
$$

Then $R_{n}:=\widehat{G}_{n}+h_{n} G_{n} \in \Sigma\left(\widehat{b}_{n}+h_{n} b_{n}\right)$, for all $n<\omega$. Moreover

$$
G=\lim _{n \rightarrow \omega} \frac{R_{n}-\widehat{G}_{n}}{h_{n}},
$$

and consequently,

$$
\begin{aligned}
y & =G\left(b_{*}\right)+G_{*}(z) \\
& =\lim _{n \rightarrow \omega} \frac{R_{n}-\widehat{G}_{n}}{h_{n}}\left(\widehat{b}_{n}\right)+R_{n}\left(b_{n}\right) \\
& =\lim _{n \rightarrow \omega} \frac{R_{n}\left(\widehat{b}_{n}+h_{n} b_{n}\right)-\widehat{G}_{n}\left(\widehat{b}_{n}\right)}{h_{n}} .
\end{aligned}
$$

Therefore, $(z, y) \in P_{\operatorname{Graph}(\check{\Sigma})}^{\check{L}}\left(b_{*}, G_{*}\left(b_{*}\right)\right)$, and consequently $y$

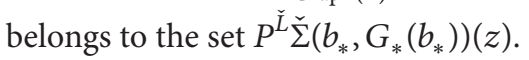

Let us begin now the last part of the proof. In this, the paraderivability of $\check{\Sigma}$ relative $\check{L}$ at $\left(b_{*}, G_{*}\left(b_{*}\right)\right) \in \check{L}$ will be shown; that is, it will be proved the inclusion

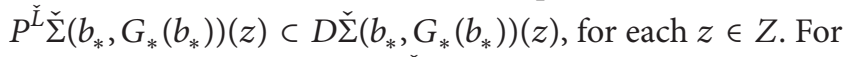
this goal let us fix $(z, y) \in P_{\operatorname{Graph}(\check{L})}^{\check{L}}\left(b_{*}, G_{*}\left(b_{*}\right)\right)$, the equality (14) and the paraderivability of $\Sigma$ relative to $L$ at $\left(b_{*}, G_{*}\right) \in L$ yield that $y \in D \Sigma\left(b_{*}, G_{*}\right)(z)\left(b_{*}\right)+G_{*}(z)$. Then there exists $G \in D \Sigma\left(b_{*}, G_{*}\right)(z)$ such that $y=G\left(b_{*}\right)+G_{*}(z)$. Hence, $\exists Z \times \mathscr{B}(Z, Y) \ni\left(b_{n}, G_{n}\right) \rightarrow(z, G)$ and $h_{n} \rightarrow 0^{+}$such that $\left(b_{*}, G_{*}\right)+h_{n}\left(b_{n}, G_{n}\right) \in \operatorname{Graph}(\Sigma)$, for every $n<\omega$. Let us define now $\widehat{G}_{n}:=G_{*}+h_{n} G_{n} \in \Sigma\left(b_{*}+h_{n} b_{n}\right)$, then there exists $\lim _{n \rightarrow+\omega}\left(\left(\widehat{G}_{n}-G_{*}\right) / h_{n}\right)=G \in \mathscr{B}(Z, Y)$. Thus, if we define $y_{n}:=\left(\widehat{G}_{n}\left(b_{*}+h_{n} b_{n}\right)-G_{*}\left(b_{*}\right)\right) / h_{n}$, since $G_{n} \rightarrow G$, we have that $y_{n} \rightarrow y$. Finally $\left(b_{*}, G_{*}\left(b_{*}\right)\right)+h_{n}\left(b_{n}, y_{n}\right)=$ $\left(b_{*}+h_{n} b_{n}, \widehat{G}_{n}\left(b_{*}+h_{n} b_{n}\right)\right) \in \operatorname{Graph}(\check{\Sigma})$, for all $n<\omega$; therefore, the proof is over.

The following example shows that in the former result neither assumptions can be dropped.

Example 5. (i) Let one defines $\Sigma_{1}:[0,+\infty) \rightarrow \mathscr{B}(\mathbb{R}, \mathbb{R})$ by $\Sigma_{1}(x):=x$, then $\Sigma_{1}$ has property $\mathscr{T}(\operatorname{Graph}(\Sigma))$ at $\left(0, \mathbf{0}_{\mathscr{B}(\mathbb{R}, \mathbb{R})}\right)$, but neither $\Sigma_{1}$ nor $\check{\Sigma}_{1}$ is paraderivable at $\left(0, \mathbf{0}_{\mathscr{B}(\mathbb{R}, \mathbb{R})}\right)$ and $(0,0)$, respectively. (ii) Let one defines $\Sigma_{2}: \mathbb{R}_{-} \cup\{0\} \cup\left\{2^{-i}: i<\omega\right\} \rightsquigarrow$ $\mathscr{B}\left(\mathbb{R}, \ell_{\infty}\right)$ by $\Sigma_{2}(t)(x):=\mathbf{0}_{\ell_{\infty}}$ if $t \in \mathbb{R}_{-} \cup\{0\}$ and $x \in \mathbb{R}$, and for every $i<\omega$,

$$
\begin{aligned}
\Sigma_{2}\left(2^{-i}\right)(x):= & \left\{(-\frac{x}{2}, \ldots, \underbrace{-\frac{x}{2}}_{i \text { th coordinate }}, \mathbf{0}_{\ell_{\infty}}),\right. \\
& (0, \ldots, 0, \underbrace{x}_{i+1 \text {-th coordinate }}, x, \ldots)\},
\end{aligned}
$$

for every $x \in \mathbb{R}$. Then $\Sigma_{2}$ is paraderivable at $\left(0, \mathbf{0}_{\mathscr{B}\left(\mathbb{R}, \ell_{\infty}\right)}\right)$, but it does not have property $\mathscr{T}(\operatorname{Graph}(\Sigma))$ at $\left(0, \mathbf{0}_{\mathscr{B}\left(\mathbb{R}, \ell_{\infty}\right)}\right)$ and $\check{\Sigma}_{2}$ is not paraderivable at $\left(0, \mathbf{0}_{\ell_{\infty}}\right)$.

In the statement of the following result, the previously fixed notation is used and, in addition, we will consider the set $\pi \check{L}=\{(b, \pi G(b)):(b, G) \in L\}$ which is a subset of $\operatorname{Graph}(\pi \check{\Sigma})$.

Lemma 6. Let one assumes that $T \Sigma$ is a single-valued and continuously Fréchet differentiable at $b_{*} \in V$ map. If $\check{\Sigma}$ is paraderivable relative to $\check{L}$ at $\left(b_{*}, G\left(b_{*}\right)\right) \in \check{L}$, then $\pi \check{\Sigma}$ is paraderivable relative to $\pi \check{L}$ at $\left(b_{*}, \pi G\left(b_{*}\right)\right)$ and

$$
\begin{aligned}
& P^{\pi \check{L}}(\pi \check{\Sigma})\left(b_{*}, \pi G_{*}\left(b_{*}\right)\right)(z)=\pi P^{\check{L} \check{\Sigma}}\left(b_{*}, G_{*}\left(b_{*}\right)\right)(z), \\
& \forall z \in Z \text {. }
\end{aligned}
$$

Proof. In this first stage of the proof we are going to check that $\pi \check{\Sigma}$ is paraderivable relative to $\pi \check{L}$ at $\left(b_{*}, \pi G\left(b_{*}\right)\right)$. To do this, we have to show the inclusion $P_{\operatorname{Graph}(\pi \check{L} \check{L})}^{\pi \check{L}}\left(b_{*}, \pi G_{*}\left(b_{*}\right)\right) \subset$ $T_{\text {Graph( } \pi \check{\Sigma})}\left(b_{*}, \pi G_{*}\left(b_{*}\right)\right)$. For this purpose, let us fix $(z, y) \in$ $P_{\operatorname{Graph}(\pi \check{L})}^{\pi \check{L}}\left(b_{*}, \pi G_{*}\left(b_{*}\right)\right) \subset Z \times \operatorname{Ker} T$, then there exists $h_{n} \rightarrow$ $0^{+}, \pi \check{L} \ni\left(\widehat{b}_{n}, \pi \widehat{G}_{n}\left(b_{n}\right)\right) \rightarrow\left(b_{*}, \pi G_{*}\left(b_{*}\right)\right)$, and $Z \times \operatorname{Ker} T \ni$ $\left(z_{n}, y_{n}\right) \rightarrow(z, y)$ such that

$$
\left(\widehat{b}_{n}, \pi \widehat{G}_{n}\left(b_{n}\right)\right)+h_{n}\left(z_{n}, y_{n}\right) \in \operatorname{Graph}(\pi \check{\Sigma}), \quad \forall n<\omega .
$$

Therefore, for any $n<\omega$, there exists $G_{n} \in \Sigma\left(\widehat{b}_{n}+h_{n} z_{n}\right)$ such that

$$
y_{n}=\frac{\pi G_{n}\left(\widehat{b}_{n}+h_{n} z_{n}\right)-\pi \widehat{G}_{n}\left(\widehat{b}_{n}\right)}{h_{n}} \in \operatorname{Ker} T, \quad \forall n<\omega .
$$

Besides, for all $n<\omega$, the following equality holds:

$$
\begin{aligned}
\frac{G_{n}\left(\widehat{b}_{n}+h_{n} z_{n}\right)-\widehat{G}_{n}\left(\widehat{b}_{n}\right)}{h_{n}} & \\
= & \frac{\pi G_{n}\left(\widehat{b}_{n}+h_{n} z_{n}\right)-\pi \widehat{G}_{n}\left(\widehat{b}_{n}\right)}{h_{n}} \\
& +\widehat{T}^{-1}\left[\frac{T G_{n}\left(\widehat{b}_{n}+h_{n} z_{n}\right)-T \widehat{G}_{n}\left(\widehat{b}_{n}\right)}{h_{n}}\right] .
\end{aligned}
$$


Now, Lemma 11 of [4] yields that $T \check{\Sigma}$ is continuously Fréchet differentiable at $b_{*}$, which provides that

$$
\begin{aligned}
& \lim _{n \rightarrow \omega} \frac{G_{n}\left(\widehat{b}_{n}+h_{n} z_{n}\right)-\widehat{G}_{n}\left(\widehat{b}_{n}\right)}{h_{n}} \\
& =y+\widehat{T}^{-1}(T \check{\Sigma})^{\prime}\left(b_{*}, z\right) \in \operatorname{Ker} T \oplus Y_{T}=Y .
\end{aligned}
$$

Also, since for all $n<\omega$, we have $\widehat{G}_{n}\left(\widehat{b}_{n}\right)=\pi \widehat{G}_{n}\left(\widehat{b}_{n}\right)+$ $\widehat{T}^{-1}\left(T \check{\Sigma}\left(b_{n}\right)\right)$, it is followed that $\lim _{n \rightarrow \omega} \widehat{G}_{n}\left(\widehat{b}_{n}\right)=G_{*}\left(b_{*}\right)$, and then

$$
\left(z, y+\widehat{T}^{-1}(T \check{\Sigma})^{\prime}\left(b_{*}, z\right)\right) \in P_{\mathrm{Graph}(\check{\Sigma})}^{\check{L}}\left(b_{*}, G_{*}\left(b_{*}\right)\right) .
$$

Paraderivability of $\check{\Sigma}$ yields

$$
\left(z, y+\widehat{T}^{-1}(T \check{\Sigma})^{\prime}\left(b_{*}, z\right)\right) \in T_{\operatorname{Graph}(\check{\Sigma})}\left(b_{*}, G_{*}\left(b_{*}\right)\right) .
$$

Hence, there exist $\bar{h}_{n} \rightarrow 0^{+}$and $Z \times Y \ni\left(\bar{z}_{n}, \bar{y}_{n}\right) \rightarrow(z, y+$ $\left.\widehat{T}^{-1}(T \check{\Sigma})^{\prime}\left(b_{*}, z\right)\right)$ such that

$$
\left(b_{*}, G_{*}\left(b_{*}\right)\right)+h_{n}\left(\bar{z}_{n}, \bar{y}_{n}\right) \in \operatorname{Graph}(\check{\Sigma}), \quad \forall n<\omega .
$$

Therefore,

$$
\left(b_{*}, \pi G_{*}\left(b_{*}\right)\right)+h_{n}\left(\bar{z}_{n}, \pi \bar{y}_{n}\right) \in \operatorname{Graph}(\pi \check{\Sigma}), \quad \forall n<\omega .
$$

Now, $y \in \operatorname{Ker} T$ implies $\lim _{n \rightarrow \omega} \pi \bar{y}_{n}=\pi y+\pi \widehat{T}^{-1}(T \check{\Sigma})^{\prime}$ $\left(b_{*}, z\right)=\pi y=y$, and then $(z, y) \in T_{\operatorname{Graph}(\pi \check{\Sigma})}\left(b_{*}, \pi G_{*}\left(b_{*}\right)\right)$.

At this point, we have shown the paraderivability of $\pi \check{\Sigma}$ and the inclusion

$$
P^{\pi \check{L}}(\pi \check{\Sigma})\left(b_{*}, \pi G_{*}\left(b_{*}\right)\right)(z) \subset \pi P^{\check{L} \check{\Sigma}}\left(b_{*}, G_{*}\left(b_{*}\right)\right)(z),
$$

$\forall z \in Z$

The proof of the lemma will be over by showing the reverse of the former inclusion. Let us fix now $y \in$

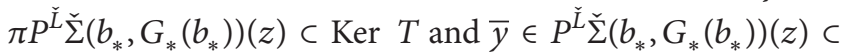
$Y$ such that $y=\pi \bar{y}$. Let us consider $h_{n} \rightarrow 0^{+}, \check{L} \ni$ $\left(b_{n}, G_{n}\left(b_{n}\right)\right) \rightarrow\left(b_{*}, G_{*}\left(b_{*}\right)\right)$ and $Z \times Y \ni\left(z_{n}, \bar{y}_{n}\right) \rightarrow(z, \bar{y})$ such that

$$
\left(b_{n}, G_{n}\left(b_{n}\right)\right)+h_{n}\left(z_{n}, \bar{y}_{n}\right) \in \operatorname{Graph}(\check{\Sigma}), \quad \forall n<\omega .
$$

Hence,

$$
\left(b_{n}, \pi G_{n}\left(b_{n}\right)\right)+h_{n}\left(z_{n}, \pi \bar{y}_{n}\right) \in \operatorname{Graph}(\pi \check{\Sigma}), \quad \forall n<\omega,
$$

and $\lim _{n \rightarrow \omega} \pi \bar{y}_{n}=\pi \bar{y}=y$. Then $y \in P^{\pi \check{L}}(\pi \check{\Sigma})\left(b_{*}, \pi G_{*}\left(b_{*}\right)\right)$ $(z)$ which finishes the proof.

Now we begin the second part of this section with a consequence of the medium value theorem. It shows the advantage of working with $\mathscr{C}^{1}$-class maps.
Lemma 7. Let $f: V \subset Z \rightarrow Y$ be a continuously Fréchet differentiable map, $V \ni b_{n} \rightarrow b_{*} \in V, Z \ni z_{n} \rightarrow z_{*} \neq 0$, and $h_{n} \rightarrow 0^{+}$. Then

$$
\lim _{n \rightarrow \omega} \frac{f\left(b_{n}+h_{n} z_{n}\right)-f\left(b_{n}\right)}{h_{n}}=f^{\prime}\left(b_{*}, z_{*}\right) .
$$

Proof. Firstly let us define the auxiliary map $g(v):=f(v)-$ $f^{\prime}\left(b_{*}, v\right)$, for every $v \in V$. Then it is also continuously Fréchet differentiable and $g^{\prime}\left(b_{*}, z\right)=0$, for every $z \in Z$. Hence, given $\epsilon>0$, there exists a neighbourhood $U$ of $b_{*}$ such that

$$
\left\|g^{\prime}(u, \cdot)\right\| \leq \frac{\epsilon}{\left\|z_{*}\right\|}, \quad \forall u \in U .
$$

Now, applying the medium value theorem, we can write

$$
0 \leq \lim _{n \rightarrow \omega}\left\|\frac{g\left(b_{n}+h_{n} z_{n}\right)-g\left(b_{n}\right)}{h_{n}}\right\| \leq \lim _{n \rightarrow \omega} \frac{\epsilon}{\left\|z_{*}\right\|} \frac{h_{n}\left\|z_{n}\right\|}{h_{n}}=\epsilon .
$$

Since $\epsilon>0$ was previously arbitrarily fixed, then

$$
\lim _{n \rightarrow \omega} \frac{g\left(b_{n}+h_{n} z_{n}\right)-g\left(b_{n}\right)}{h_{n}}=0 .
$$

In fact, the former limit allows us to compute the limit of the statement because

$$
\begin{aligned}
\lim _{n \rightarrow \omega} & {\left[\frac{f\left(b_{n}+h_{n} z_{n}\right)-f\left(b_{n}\right)}{h_{n}}-f^{\prime}\left(b_{*}, z_{*}\right)\right] } \\
= & \lim _{n \rightarrow \omega} \frac{f\left(b_{n}+h_{n} z_{n}\right)-f\left(b_{n}\right)}{h_{n}}-\lim _{n \rightarrow \omega} f^{\prime}\left(b_{*}, z_{n}\right) \\
= & \lim _{n \rightarrow \omega} \frac{f\left(b_{n}+h_{n} z_{n}\right)-f\left(b_{n}\right)}{h_{n}} \\
& -\lim _{n \rightarrow \omega} \frac{f^{\prime}\left(b_{*}, b_{n}+h_{n} z_{n}\right)-f^{\prime}\left(b_{*}, b_{n}\right)}{h_{n}} \\
= & \lim _{n \rightarrow \omega} \frac{g\left(b_{n}+h_{n} z_{n}\right)-g\left(b_{n}\right)}{h_{n}}=0,
\end{aligned}
$$

and the proof is over.

In the following result we see how a condition in form of inclusion for a set-valued map $\Sigma: V \subset Z \leadsto \mathscr{B}(Z, Y)$ allows us to turn some pointwise limits, of linear and continuous maps, into uniform ones in a stronger way than property $\mathscr{T}$ does. It will be useful in the proof of the main theorem of the work, in Section 4.

Proposition 8. Let $\alpha: V \subset Z \rightarrow \mathscr{B}(Z, Y)$ and $\beta: V \subset$ $Z \rightarrow Z^{*}$ be two continuously Fréchet differentiable maps such that $\beta(b)(b)=1$ for every $b \in V$. Let one fixes also a set-valued map $\Sigma: V \leadsto \mathscr{B}(Z, Y)$ such that for every $b \in V \Sigma(b) \subset\{\alpha(b)+y \beta(b): y \in Y\}$, a point $\left(b_{*}, G_{*}\right) \in L \subset$ $\operatorname{Graph}(\Sigma)$, and four sequences: $Z \ni b_{n} \rightarrow z, h_{n} \rightarrow 0^{+}$, 
$\check{L} \ni\left(\widehat{b}_{n}, \widehat{G}_{n}\left(\widehat{b}_{n}\right)\right) \rightarrow\left(b_{*}, G_{*}\left(b_{*}\right)\right)$, and $\left\{G_{n}\right\}_{n<\omega} \subset \mathscr{B}(Z, Y)$ such that each $G_{n} \in \sum\left(\widehat{b}_{n}+h_{n} b_{n}\right)$. Now, if there exists

$$
\lim _{n \rightarrow \omega} \frac{G_{n}\left(\widehat{b}_{n}+h_{n} b_{n}\right)-\widehat{G}_{n}\left(\widehat{b}_{n}\right)}{h_{n}} \in Y,
$$

then there exists

$$
\lim _{n \rightarrow \omega} \frac{G_{n}-\widehat{G}_{n}}{h_{n}} \in \mathscr{B}(Z, Y) \text { as well. }
$$

Proof. In the first place, the inclusion of $\Sigma(b)$ in the statement yields that there are $y_{*} \in Y$ and two sequences $\left\{y_{n}\right\}_{n<\omega}$ and $\left\{\hat{y}_{n}\right\}_{n<\omega}$ of elements of $Y$ such that $G_{*}=\alpha\left(b_{*}\right)+y_{*} \beta\left(b_{*}\right)$, $G_{n}=\alpha\left(\widehat{b}_{n}+h_{n} b_{n}\right)+y_{n} \beta\left(\widehat{b}_{n}+h_{n} b_{n}\right)$, and $\widehat{G}_{n}=\alpha\left(\widehat{b}_{n}\right)+\widehat{y}_{n} \beta\left(\widehat{b}_{n}\right)$ for every $n<\omega$.

Now, on the one hand,

$$
\begin{aligned}
& \frac{G_{n}\left(\widehat{b}_{n}+h_{n} b_{n}\right)-\widehat{G}_{n}\left(\widehat{b}_{n}\right)}{h_{n}} \\
&=\frac{\alpha\left(\widehat{b}_{n}+h_{n} b_{n}\right)\left(\widehat{b}_{n}+h_{n} b_{n}\right)-\alpha\left(\widehat{b}_{n}\right)\left(\widehat{b}_{n}\right)}{h_{n}}+\frac{y_{n}-\widehat{y}_{n}}{h_{n}}, \\
& \forall n<\omega .
\end{aligned}
$$

On the other hand, since $\alpha$ is continuously Fréchet differentiable, the former lemma and Lemma 10 of [6] yield

$$
\lim _{n \rightarrow \omega} \frac{y_{n}-\widehat{y}_{n}}{h_{n}}=y-\alpha^{\prime}\left(b_{*}, z\right)\left(b_{*}\right)-\alpha\left(b_{*}\right)(z),
$$

where $z=\lim _{n \rightarrow \omega} b_{n} \in Z$ and $y=\lim _{n \rightarrow \omega}\left(\left(G_{n}\left(\widehat{b}_{n}+h_{n} b_{n}\right)-\right.\right.$ $\left.\left.\widehat{G}_{n}\left(\widehat{b}_{n}\right)\right) / h_{n}\right) \in Y$.

To conclude, for every $n<\omega$, we decompose

$$
\begin{aligned}
\frac{G_{n}-\widehat{G}_{n}}{h_{n}} & \\
= & \frac{\alpha\left(\widehat{b}_{n}+h_{n} b_{n}\right)+y_{n} \beta\left(\widehat{b}_{n}+h_{n} b_{n}\right)-\alpha\left(\widehat{b}_{n}\right)-\widehat{y}_{n} \beta\left(\widehat{b}_{n}\right)}{h_{n}} \\
= & \frac{\alpha\left(\widehat{b}_{n}+h_{n} b_{n}\right)-\alpha\left(\widehat{b}_{n}\right)}{h_{n}}+y_{n} \frac{\beta\left(\widehat{b}_{n}+h_{n} b_{n}\right)-\beta\left(\widehat{b}_{n}\right)}{h_{n}} \\
& +\frac{y_{n}-\widehat{y}_{n}}{h_{n}} \beta\left(\widehat{b}_{n}\right) .
\end{aligned}
$$

The former decomposition allows us to arrive at

$$
\begin{aligned}
\lim _{n \rightarrow \omega} \frac{G_{n}-\widehat{G}_{n}}{h_{n}} \\
=\alpha^{\prime}\left(b_{*}, z\right)+y_{*} \beta^{\prime}\left(b_{*}, z\right) \\
\quad+\left[y-\alpha^{\prime}\left(b_{*}, z\right)\left(b_{*}\right)-\alpha\left(b_{*}\right)(z)\right] \beta\left(b_{*}\right),
\end{aligned}
$$

Let us compute now the formula for the paratingent derivative of the sum of a set-valued map and a single-valued map. For the formula of the sum with other derivatives, we refer the reader to [2].

Proposition 9. Let $f: V \subset Z \rightarrow Y$ be a continuously Fréchet differentiable map, $F: V \subset Z \leadsto Y$ a set-valued map, $L \subset$ $\operatorname{Graph}(F),\left(b_{*}, y_{*}\right) \in L$, and $L^{\prime}=\{(b, f(b)+y) \in V \times Y$ : $(b, y) \in L\}$. Then

$$
\begin{aligned}
& P^{L^{\prime}}(f+F)\left(b_{*}, f\left(b_{*}\right)+y_{*}\right)(z) \\
& \quad=f^{\prime}\left(b_{*}, z\right)+P^{L} F\left(b_{*}, y_{*}\right)(z), \quad \forall z \in Z .
\end{aligned}
$$

Proof. Let us begin by checking the inclusion $\subset$. For this purpose we fix arbitrary elements $z \in Z$ and $y \in P^{L^{\prime}}(f+$ $F)\left(b_{*}, f\left(b_{*}\right)+y_{*}\right)(z)$. Now, by the usual characterization of the paratingent cone we have three sequences: $h_{n} \rightarrow 0^{+}$, $Z \times Y \ni\left(z_{n}, y_{n}\right) \rightarrow(z, y)$, and $L^{\prime} \ni\left(b_{n}, f\left(b_{n}\right)+y^{*}\right) \rightarrow$ $\left(b_{*}, f\left(b_{*}\right)+y_{*}\right)$. Moreover, the following condition holds true for every $n<\omega$ :

$$
f\left(b_{n}\right)+y_{n}^{*}+h_{n} y_{n} \in f\left(b_{n}+h_{n} z_{n}\right)+F\left(b_{n}+h_{n} z_{n}\right) .
$$

Then, for every $n<\omega$, there exists $\widehat{z}_{n} \in F\left(b_{n}+h_{n} z_{n}\right)$ such that

$$
y_{n}=\frac{f\left(b_{n}+h_{n} z_{n}\right)-f\left(b_{n}\right)}{h_{n}}+\frac{\widehat{z}_{n}-y_{n}^{*}}{h_{n}} \in Y,
$$

which yields

$$
\lim _{n \rightarrow \omega} \frac{\widehat{z}_{n}-y_{n}^{*}}{h_{n}}=y-f^{\prime}\left(b_{*}, z\right) .
$$

Finally, the definition of paratingent cone assures that the last limit belongs to $P^{L} F\left(b_{*}, y_{*}\right)(z)$, and hence, $y-f^{\prime}\left(b_{*}, z\right)$ also does.

In order to prove the reverse inclusion, we fix arbitrary $z \in Z$ and $y \in f^{\prime}\left(b_{*}, z\right)+P^{L} F\left(b_{*}, y_{*}\right)(z)$. We use again the characterization of paratingent cones which provides us the usual three sequences: $h_{n} \rightarrow 0^{+}, Z \times Y \ni\left(z_{n}, y_{n}\right) \rightarrow(z, y-$ $\left.f^{\prime}\left(b_{*}, z\right)\right)$, and $L \ni\left(b_{n}, y_{n}^{*}\right) \rightarrow\left(b_{*}, y_{*}\right)$ in such away that

$$
y_{n}^{*}+h_{n} y_{n} \in F\left(b_{n}+h_{n} z_{n}\right), \quad \forall n<\omega .
$$

Now, for every $n<\omega$, there exists $\widehat{z}_{n} \in F\left(b_{n}+h_{n} z_{n}\right)$ such that $y_{n}=\left(\widehat{z}_{n}-y_{n}^{*}\right) / h_{n}$, and the sequence defined by the general term

$$
w_{n}=\frac{f\left(b_{n}+h_{n} z_{n}\right)+\widehat{z}_{n}-f\left(b_{n}\right)-y_{n}^{*}}{h_{n}} \in Y
$$

converges to $y$. To conclude, since

$$
\left(b_{n}, f\left(b_{n}\right)+y_{n}^{*}\right)+h_{n}\left(z_{n}, w_{n}\right) \in \operatorname{Graph}(f+F), \quad \forall n<\omega,
$$

certainly

$$
y \in P^{L^{\prime}}(f+F)\left(b_{*}, f\left(b_{*}\right)+y_{*}\right)(z) .
$$

and thus, the proof is over. 


\section{Sensitivity Analysis}

Theorem A will be proved in this section. However, before this, it will be necessary to state and prove Theorem 12. In this theorem, it is shown how the Fréchet differentiability of the single-valued map $T \Psi$ allows us to transfer the paraderivability from the $T$-dual perturbation map $\Psi$ to the set-valued map $\check{\Psi}$. In its proof, the obtained results in Section 3 are applied, taking $\Sigma$ as $\Psi$. In addition, we will need to establish also some previous technical results which will constitute the first part of this section.

Throughout this section we will assume, firstly, that the parameter $b$ belongs to an open convex set $V \subset Z$ such that $0 \notin V$. This condition is not a restriction because the problem (1) with $b=0$ is equivalent to some problem (1) with $b \neq 0$. If we keep this assumption in mind, the following claim can be proved. There exists a continuously Fréchet differentiable $\operatorname{map} \beta: V \rightarrow Z^{*}$ such that $\beta(v)(v)=1$ for each $v \in V$. This is a consequence of the Hanh-Banach theorem, Theorem 3.4 of [28].

In the second place, we will assume that for every $b \in \mathrm{V}$ there exists a regular local $T$-optimal solution $x_{b} \in D$ of (1) in such a way that the map $\lambda: V \rightarrow X$ given by $\lambda(b)=x_{b}$ is Fréchet differentiable. The existence of this kind of map has been studied by several authors; the linear case can be seen in [30]. This assumption jointly with Proposition 8 of [6] implies that $T \Psi$ is a single-valued map, that is, $T G=T G^{\prime} \in \mathscr{B}(Z, W)$ for every two elements $G, G^{\prime} \in \Psi(b)$ and $b \in V$.

Definition 10. Let $b \in V, x_{b} \in D$ a regular local $T$-optimal solution of (1), and $G_{x_{b}} \in \mathscr{B}(Z, Y)$ a $T$-Lagrange multiplier of (1) associated with $x_{b}$. The $(T, \beta)$-modification of $G_{x_{b}}$ is the map defined by

$$
G_{x_{b}}^{(T, \beta)}(z):=\widehat{T}^{-1} T G_{x_{b}}(z)+\pi G_{x_{b}}(b) \beta(b)(z), \quad \forall z \in Z .
$$

It is easy to check that $G_{x_{b}}^{(T, \beta)}$ is a $T$-Lagrange multiplier of (1) associated with $x_{b}$. Moreover, $(T, \beta)$-modifications of two different $T$-Lagrange multipliers of (1) associated to the same regular local $T$-optimal solution coincide. Moreover, we have the following result.

Proposition 11 (see Proposition 10 of [19]). Let $b \in V, x_{b} \in D$ a regular local $T$-optimal solution of (1), $G_{x_{b}} \in \mathscr{B}(Z, Y)$ a $T$ Lagrange multiplier of (1) associated with $x_{b}$, and $\mathcal{U}_{x_{b}}$ the set of the T-Lagrange multipliers of (1) associated with $x_{b}$. Then

$$
\mathscr{U}_{x_{b}}=\left\{G_{x_{b}}^{(T, \beta)}\right\}+\mathscr{H}_{b},
$$

where $\mathscr{H}_{b}=\{H \in \mathscr{B}(Z, \operatorname{Ker} T): H(b)=0\}$.

Next we will prove the following.

Theorem 12. Let one supposes that the T-dual perturbation map $\Psi$ is paraderivable relative to $L$ at $\left(b_{*}, G_{*}\right) \in L \subset$ $\operatorname{Graph}(\Psi)$ and $T \Psi$ is Fréchet differentiable at $b_{*} \in V$. Then $\check{\Psi}$ is paraderivable relative to

$$
\check{L}:=\{(b, G(b)) \in Z \times Y:(b, G) \in L\}
$$

$$
\begin{aligned}
& \text { at }\left(b_{*}, G_{*}\left(b_{*}\right)\right) \text { and } \\
& \qquad \begin{array}{l}
P^{\check{L} \check{\Psi}}\left(b_{*}, G_{*}\left(b_{*}\right)\right)(z) \\
\quad=P^{L} \Psi\left(b_{*}, G_{*}\right)(z)\left(b_{*}\right)+G_{*}(z), \quad \forall z \in Z .
\end{array}
\end{aligned}
$$

Proof. The statement will be proved by applying Theorem 4 . Hence, it is enough to prove that $\Psi$ verifies property $\mathscr{T}(L)$ at $\left(b_{*}, G_{*}\right) \in L$. For this, let us fix the four sequences which appear in the first part of the definition of Definition 3, that is, $h_{n} \rightarrow 0^{+}, Z \ni b_{n} \rightarrow b \in Z, \check{L} \ni\left(\widehat{b}_{n}, \widehat{G}_{n}\left(\widehat{b}_{n}\right)\right) \rightarrow\left(b_{*}, G_{*}\left(b_{*}\right)\right)$, and $\left\{G_{n}\right\}_{n<\omega} \subset \mathscr{B}(Z, Y)$ such that each $G_{n} \in \Sigma\left(\widehat{b}_{n}+h_{n} b_{n}\right)$ and the below limit exists

$$
\lim _{n \rightarrow \omega} \frac{G_{n}\left(\widehat{b}_{n}+h_{n} b_{n}\right)-\widehat{G}_{n}\left(\widehat{b}_{n}\right)}{h_{n}} \in Y .
$$

Now we can consider, on the one hand, the continuously Fréchet differentiable map $\beta: V \rightarrow Z^{*}$ defined at the beginning of this section and such that $\beta(b)(b)=1$ for every $b \in V$. On the other hand, we consider the auxiliary maps $G_{n}^{(T, \beta)}$ and $\widehat{G}_{n}^{(T, \beta)}$ for every $n<\omega$. By Proposition 11 we have

$$
\begin{aligned}
G_{n}\left(\widehat{b}_{n}+h_{n} b_{n}\right) & =G_{n}^{(T, \beta)}\left(\widehat{b}_{n}+h_{n} b_{n}\right), \\
\widehat{G}_{n}\left(\widehat{b}_{n}\right) & =\widehat{G}_{n}^{(T, \beta)}\left(\widehat{b}_{n}\right),
\end{aligned}
$$

for every $n<\omega$. Moreover, the below limit exists

$$
\lim _{n \rightarrow \omega} \frac{G_{n}^{(T, \beta)}\left(\widehat{b}_{n}+h_{n} b_{n}\right)-\widehat{G}_{n}^{(T, \beta)}\left(\widehat{b}_{n}\right)}{h_{n}} \in Y .
$$

Then Proposition 8 applied to the set-valued map

$$
\begin{aligned}
\Psi^{(T, \beta)}: V & \rightsquigarrow \mathscr{B}(Z, Y), \\
b & \rightsquigarrow \Psi^{(T, \beta)}(b)=\left\{G^{(T, \beta)} \in \mathscr{B}(Z, Y): G \in \Psi(b)\right\}
\end{aligned}
$$

implies that the following also exists:

$$
\lim _{n \rightarrow \omega} \frac{G_{n}^{(T, \beta)}-\widehat{G}_{n}^{(T, \beta)}}{h_{n}} \in \mathscr{B}(Z, Y) .
$$

Now, we have all necessary ingredients at hand in order to check that $\Psi$ enjoys property $\mathscr{T}(L)$ at $\left(b_{*}, G_{*}\right) \in L$. For this purpose, we will define three sequences which verify the conditions stated in the second part of Definition 3. In fact, for each $n<\omega$, we consider $\bar{b}_{n}:=b_{n}$, and define the maps $\widetilde{G}_{n}$ and $\bar{G}_{n}$ by

$$
\begin{aligned}
\widetilde{G}_{n}(z):= & \widehat{G}_{n}^{(T, \beta)}(z)+\left(G_{*}-G_{*}^{(T, \beta)}\right)(z) \\
& +\left(G_{*}^{(T, \beta)}-G_{*}\right)\left(\widehat{b}_{n}\right) \beta\left(\widehat{b}_{n}\right)(z), \\
\bar{G}_{n}(z):= & G_{n}^{(T, \beta)}(z)+\left(G_{*}-G_{*}^{(T, \beta)}\right)(z) \\
& +\left(G_{*}^{(T, \beta)}-G_{*}\right)\left(\widehat{b}_{n}+h_{n} b_{n}\right) \beta\left(\widehat{b}_{n}+h_{n} b_{n}\right)(z),
\end{aligned}
$$


for every $z \in Z$. Clearly $\left\{\bar{G}_{n}\right\}_{n<\omega},\left\{\widetilde{G}_{n}\right\}_{n<\omega} \subset \mathscr{B}(Z, Y)$. Again Proposition 11 yields $\bar{G}_{n} \in \Psi\left(\widehat{b}_{n}+h_{n} b_{n}\right)$ and $\widetilde{G}_{n} \in \Psi\left(\widehat{b}_{n}\right)$ for every $n<\omega$. Furthermore, since each $\bar{G}_{n}\left(\widehat{b}_{n}+h_{n} b_{n}\right)=$ $G_{n}^{(T, \beta)}\left(\widehat{b}_{n}+h_{n} b_{n}\right)$ and $\widetilde{G}_{n}\left(\widehat{b}_{n}\right)=\widehat{G}_{n}^{(T, \beta)}\left(\widehat{b}_{n}\right)$, from $(60)$ we get $\bar{G}_{n}\left(\widehat{b}_{n}+h_{n} b_{n}\right)=G_{n}\left(\widehat{b}_{n}+h_{n} b_{n}\right)$ and $\widetilde{G}_{n}\left(\widehat{b}_{n}\right)=\widehat{G}_{n}\left(\widehat{b}_{n}\right)$. This provides the equality

$$
\begin{aligned}
& \lim _{n \rightarrow \omega} \frac{\bar{G}_{n}\left(\widehat{b}_{n}+h_{n} \bar{b}_{n}\right)-\widetilde{G}_{n}\left(\widehat{b}_{n}\right)}{h_{n}} \\
&=\lim _{n \rightarrow \omega} \frac{G_{n}\left(\widehat{b}_{n}+h_{n} b_{n}\right)-\widehat{G}_{n}\left(\widehat{b}_{n}\right)}{h_{n}} \in Y .
\end{aligned}
$$

The last step in the proof is to check that $\lim _{n \rightarrow \omega}\left(\bar{G}_{n}-\widetilde{G}_{n}\right) / h_{n}$ exists. Indeed, for every $n<\omega$,

$$
\begin{aligned}
\frac{\bar{G}_{n}-\widetilde{G}_{n}}{h_{n}} & \\
= & \frac{G_{n}^{(T, \beta)}-\widehat{G}_{n}^{(T, \beta)}}{h_{n}} \\
& +\left(G_{*}-G_{*}^{(T, \beta)}\right)\left(\widehat{b}_{n}\right) \frac{\beta\left(\widehat{b}_{n}+h_{n} b_{n}\right)-\beta\left(\widehat{b}_{n}\right)}{h_{n}} \\
& +\left(G_{*}-G_{*}^{(T, \beta)}\right)\left(b_{n}\right) \beta\left(\widehat{b}_{n}+h_{n} b_{n}\right) .
\end{aligned}
$$

Now, taking into account that $\beta$ is continuously differentiable at $b_{*}$, from Lemma 7 , we obtain that

$$
\lim _{n \rightarrow \omega} \frac{\beta\left(\widehat{b}_{n}+h_{n} b_{n}\right)-\beta\left(\widehat{b}_{n}\right)}{h_{n}}=\beta^{\prime}\left(b_{*}, b\right) .
$$

Therefore,

$$
\begin{aligned}
& \lim _{n \rightarrow \omega} \frac{\bar{G}_{n}-\widetilde{G}_{n}}{h_{n}} \\
& =\lim _{n \rightarrow \omega} \frac{G_{n}^{(T, \beta)}-\widehat{G}_{n}^{(T, \beta)}}{h_{n}}+\left(\widehat{G}_{*}-\widehat{G}_{*}^{(T, \beta)}\right)\left(b_{*}\right) \beta^{\prime}\left(b_{*}, b\right) \\
& \quad+\left(G_{*}-G_{*}^{(T, \beta)}\right)(b) \beta\left(b_{*}\right),
\end{aligned}
$$

and the proof is over.

Now we can prove Theorem A.

Proof of Theorem A. In the first part of the proof we will check that $\pi \Upsilon$ is paraderivable relative to $\pi \check{L}_{\Psi}$ at $\left(b_{*}, \pi f\left(x_{b_{*}}\right)\right)$. After that we will finish the proof by means of the equality

$$
\Upsilon(b)=\widehat{T}^{-1} T \Upsilon(b)+\pi \Upsilon(b), \quad \forall b \in V .
$$

Since $\pi \Upsilon(b)=\pi \check{\Psi}(b)$ for every $b \in V$, it is enough to check that the set-valued map $\pi \check{\Psi}$ is paraderivable relative to $\pi \check{L}_{\Psi}$ at $\left(b_{*}, \pi G_{x_{b_{*}}}\left(b_{*}\right)\right)$. Applying Theorem 12 we get that $\check{\Psi}$ is paraderivable relative to

$$
\check{L}_{\Psi}=\left\{(b, G(b)) \in Z \times X:(b, G) \in L_{\Psi}\right\},
$$

at $\left(b_{*}, G_{x_{b_{*}}}\left(b_{*}\right)\right)$ and

$$
\begin{aligned}
P^{\check{L}} \Psi & \check{\Psi}\left(b_{*}, G_{x_{b_{*}}}\left(b_{*}\right)\right)(z) \\
& =P^{L_{\Psi}} \Psi\left(b_{*}, G_{x_{b_{*}}}\right)(z)\left(b_{*}\right)+G_{x_{b_{*}}}(z), \quad \forall z \in Z .
\end{aligned}
$$

By Lemma $6 \pi \check{\Psi}$ is paraderivable relative to $\pi \check{L}_{\Psi}$ at $\left(b_{*}, \pi G_{x_{b_{*}}}\left(b_{*}\right)\right)$ and

$$
\begin{aligned}
\pi P^{\check{L}_{\Psi}} \check{\Psi}\left(b_{*}, G_{x_{b_{*}}}\left(b_{*}\right)\right)(z) \\
=P^{\pi \check{L}_{\Psi}} \pi \check{\Psi}\left(b_{*}, \pi G_{x_{b_{*}}}\left(b_{*}\right)\right)(z), \quad \forall z \in Z .
\end{aligned}
$$

Now, the equality $\pi \Upsilon=\pi \check{\Psi}$ and (71) yield

$$
\begin{aligned}
& P^{\pi \check{L}_{\Psi}} \pi \Upsilon\left(b_{*}, \pi f\left(x_{b_{*}}\right)\right)(z) \\
& \quad=\pi G_{x_{b_{*}}}(z)+\pi P^{L_{\Psi}} \Psi\left(b_{*}, G_{x_{b_{*}}}\right)(z)\left(b_{*}\right), \quad \forall z \in Z .
\end{aligned}
$$

In order to finish the proof we consider the equality (69). On the one hand, by Proposition 8 of [6], the function $T \Upsilon$ is continuously Fréchet differentiable at $b_{*}$ and $[T \Upsilon]^{\prime}\left(b_{*}, z\right)=$ $T G_{x_{b_{*}}}(z)$, for every $z \in Z$. Thus, $\left[\widehat{T}^{-1} T \Upsilon\right]^{\prime}\left(b_{*}, z\right)=$ $\widehat{T}^{-1} T G_{x_{b_{*}}}(z)$ for every $z \in Z$. On the other hand, if we apply Proposition 9 to equality (69), we obtain that the set-valued map $\Upsilon$ is paraderivable relative to $L_{\Upsilon}$ at $\left(b_{*}, f\left(x_{b_{*}}\right)\right)$ and

$$
\begin{aligned}
P^{L_{\Upsilon}} & \Upsilon\left(b_{*}, f\left(x_{b_{*}}\right)\right)(z) \\
& =\widehat{T}^{-1} T G_{x_{b_{*}}}(z)+\pi G_{x_{b_{*}}}(z)+\pi P^{L_{\Psi}} \Psi\left(b_{*}, G_{x_{b_{*}}}\right)(z)\left(b_{*}\right) \\
& =G_{x_{b_{*}}}(z)+\pi P^{L_{\Psi}} \Psi\left(b_{*}, G_{x_{b_{*}}}\right)(z)\left(b_{*}\right), \quad \forall z \in Z .
\end{aligned}
$$

This section is finished by illustrating Theorem A with the aid of an example.

Example 13. Let one considers $X=Y=\mathbb{R}^{3}, Z=W=\mathbb{R}$, the interval $V=(-11 / 10,9 / 10) \subset \mathbb{R}, D=\left\{(x, y, z) \in \mathbb{R}^{3}\right.$ : $\left.2 \cosh y^{2}>z\right\}$, and the problem

$$
\begin{gathered}
\operatorname{Min}(x-\sqrt{2} y+z, x+\sqrt{2} y+z,-\sqrt{2} x+\sqrt{2} z) \\
-\log \left(2 \cosh y^{2}-z\right)=b, \quad(x, y, z) \in D,
\end{gathered}
$$

for every $b \in V$.

Let us take $T=(1,1, \sqrt{2})$. Solving the problem we obtain the $T$-perturbation map

$$
\begin{aligned}
\Upsilon(b)=\{( & \left(\frac{2 e^{b}-1}{e^{b}}-\lambda, \frac{2 e^{b}-1}{e^{b}}-\lambda, \sqrt{2} \frac{2 e^{b}-1}{e^{b}}+\sqrt{2} \lambda\right) \\
& \left.\in \mathbb{R}^{3}: \lambda \in \mathbb{R}\right\},
\end{aligned}
$$

for every $b \in V$. 
Let us study the sensitivity of the problem (75) at $b=-1$, the point $x_{-1}=(0,0,2-e)$, and so $f\left(x_{1}\right)=(2-e, 2-$ $e, \sqrt{2}(2-e))$, taking $L=\operatorname{Graph}(\Upsilon)$. We first analyze directly the sensitivity by calculating

$$
\begin{aligned}
P Y & \left(-1, f\left(x_{-1}\right)\right)(u) \\
& =\{(e u+\lambda, e u+\lambda, \sqrt{2} e u-\sqrt{2} \lambda): \lambda \in \mathbb{R}\}, \quad \forall u \in \mathbb{R} .
\end{aligned}
$$

Let us now apply Theorem A to verify (77). Since Ker $T$ is the linear space generated by the vectors $(-\sqrt{2}, 0,1)$ and $(-1,1,0)$, taking $Y_{T}=(\operatorname{Ker} T)^{\perp}$ we obtain $G_{x_{-1}}(u)=$ (eu, eu, $\sqrt{2} e u)$ and the $T$-dual perturbation map

$$
\Psi(b)=\left\{\left(e^{-b}+\frac{\lambda}{b}, e^{-b}+\frac{\lambda}{b}, \sqrt{2} e^{-b}-\frac{\sqrt{2} \lambda}{b}\right): \lambda \in \mathbb{R}\right\} .
$$

Thus, we have

$$
\begin{aligned}
& P \Psi\left(-1, G_{x_{-1}}\right)(u)(b) \\
& =\left\{\left(-e^{-b} u-\frac{\lambda}{b^{2}},-e^{-b} u-\frac{\lambda}{b^{2}},-\sqrt{2} e^{-b} u+\frac{\sqrt{2} \lambda}{b^{2}}\right): \lambda \in \mathbb{R}\right\},
\end{aligned}
$$

for every $u \in \mathbb{R}$, and therefore

$$
\pi P \Psi\left(-1, G_{x_{-1}}\right)(u)(-1)=\{(-\lambda,-\lambda, \sqrt{2} \lambda): \lambda \in \mathbb{R}\} .
$$

Finally we obtain that

$$
\begin{aligned}
G_{x_{-1}}(u)+\pi P \Psi\left(-1, G_{x_{-1}}\right)(u)(-1) \\
=(e u, e u, \sqrt{2} e u)+\{(-\lambda,-\lambda, \sqrt{2} \lambda): \lambda \in \mathbb{R}\} \\
=\{(e u-\lambda, e u-\lambda, \sqrt{2} e u+\sqrt{2} \lambda): \lambda \in \mathbb{R}\} \\
=P \Upsilon\left(-1, f\left(x_{-1}\right)\right)(u)
\end{aligned}
$$

Consequently, equality (4) holds as Theorem A states.

\section{Concluding Remarks}

The objective of this article is to analyze the sensitivity of a multiobjective differential program with equality constraints. Given a family of parameterized programs, the $T$ perturbation set-valued map is defined as the correspondence that assigns to each right-hand side parameter value the set of minimal points of its associated program, on which a positive linear continuous map, $T$, takes a minimum value. The behavior of the $T$-perturbation map is analyzed quantitatively by making use of the paratingent derivative. The main result of the paper is stated in Theorem A, where we assert that the sensitivity of the program is measured by a Lagrange multiplier plus the projection of its derivative onto the kernel of $T$. The use of the paratingent derivative in the analysis transmits to the obtained result its distinctive stability with respect to disturbances around the point where the analysis is performed. In previous papers, some authors have carried out this kind of analysis by means of other derivatives. By doing so, this research completes the study of sensitivity of $T$-optimal solutions of this program by means of the four main set-valued derivatives, that is, contingent, adjacent, circatangent, and paratingent derivatives.

\section{Acknowledgment}

F. García was partially supported by the Universidad de Alicante Project GRE11-08.

\section{References}

[1] B. S. Mordukhovich, Variational Analysis and Generalized Differentiation II. Applications, vol. 331 of A Series of Comprehensive Studies in Mathematics, Springer, Berlin, Germany, 2006.

[2] J.-P. Aubin and H. Frankowska, Set-Valued Analysis, Birkhäuser, Boston, Mass, USA, 1990.

[3] J.-P. Aubin and H. Frankowska, Controlability and Observability of Control Systems under Uncertainty, International Institute for Applied Systems Analysis, Vienna, Austria, 1989.

[4] A. Balbás and P. Jiménez Guerra, "Sensitivity analysis for convex multiobjective programming in abstract spaces," Journal of Mathematical Analysis and Applications, vol. 202, no. 2, pp. 645658, 1996.

[5] A. Balbás and P. Jiménez Guerra, "Sensitivity in multiobjective programming by differential equations methods. The case of homogeneous functions," in Advances in Multiple Objective and Goal Programming, R. Caballero and F. Ruiz, Eds., vol. 455 of Lecture Notes in Economics and Mathematical Systems, pp. 188196, Springer, Berlin, Germany, 1997.

[6] A. Balbás, M. E. Ballve, and P. Jiménez Guerra, "Sensitivity and optimality conditions in the multiobjective differential programming," Indian Journal of Pure and Applied Mathematics, vol. 29, no. 7, pp. 671-680, 1998.

[7] A. Balbás, M. Ballvé, P. Jiménez Guerra et al., "Sensitivity in multiobjective programming under homogeneity assumptions," Journal of Multi-Criteria Decision Analysis, vol. 8, no. 3, pp. 133-138, 1999.

[8] A. Balbás, F. J. Fernández, and P. Jiménez Guerra, "On the envolvent theorem in multiobjective programming," Indian Journal of Pure and Applied Mathematics, vol. 26, no. 11, pp. 1035-1047, 1995.

[9] T. Gal and K. Wolf, "Stability in vector maximization. A survey," European Journal of Operational Research, vol. 25, no. 2, pp. 169182, 1986.

[10] F. García and M. A. Melguizo, "Sensitivity analysis in convex optimization through the circatangent derivative," submitted to Journal of Optimization Theory and Applications.

[11] P. Jiménez Guerra, M. A. Melguizo, and M. J. Muñoz-Bouzo, "Sensitivity analysis in convex programming," Computers \& Mathematics with Applications, vol. 58, no. 6, pp. 1239-1246, 2009.

[12] T. Tanino, "Sensitivity analysis in multiobjective optimization," Journal of Optimization Theory and Applications, vol. 56, no. 3, pp. 479-499, 1988.

[13] T. Tanino, "Stability and sensitivity analysis in convex vector optimization," SIAM Journal on Control and Optimization, vol. 26, no. 3, pp. 521-536, 1988. 
[14] T. Tanino, "Sensitivity analysis in MCDM," in Multicriteria Decision Making: Advances in MCDM Models, Algorithms, Theory, and Applications, T. Gal, T. Stewart, and T. Hanne, Eds., pp. 7.1-7.29, Kluwer Academic, Boston, Mass, USA, 1999.

[15] T. D. Chuong and J. C. Yao, "Generalized Clarke epiderivatives of parametric vector optimization problems," Journal of Optimization Theory and Applications, vol. 146, no. 1, pp. 77-94, 2010.

[16] T. D. Chuong, J.-C. Yao, and N. D. Yen, "Further results on the lower semicontinuity of efficient point multifunctions," Pacific Journal of Optimization, vol. 6, no. 2, pp. 405-422, 2010.

[17] T. D. Chuong, "Clarke coderivatives of efficient point multifunctions in parametric vector optimization," Nonlinear Analysis. Theory, Methods \& Applications, vol. 74, no. 1, pp. 273-285, 2011.

[18] P. Jiménez Guerra and M. A. Melguizo Padial, "Sensitivity analysis in differential programming through the Clarke derivative," Mediterranean Journal of Mathematics, vol. 9, no. 3, pp. 537-550, 2012.

[19] P. Jiménez Guerra, M. A. Melguizo, and M. J. Muñoz-Bouzo, "Sensitivity analysis in multiobjective differential programming," Computers \& Mathematics with Applications, vol. 52, no. 1-2, pp. 109-120, 2006.

[20] L. Zemin, "The optimality conditions of differentiable vector optimization problems," Journal of Mathematical Analysis and Applications, vol. 201, no. 1, pp. 35-43, 1996.

[21] J.-P. Aubin and A. Cellina, Differential Inclusions Set-Valued Maps and Viability Theory, Springer, Berlin, Germany, 1984.

[22] L. Boudjenah, "Existence of solutions to a paratingent equation with delayed argument," Electronic Journal of Differential Equations, vol. 2005, no. 14, pp. 1-8, 2005.

[23] A. Gorre, "Evolutions of tubes under operability constraints," Journal of Mathematical Analysis and Applications, vol. 216, no. 1, pp. 1-22, 1997.

[24] M.-C. Arnaud, "The link between the shape of the irrational Aubry-Mather sets and their Lyapunov exponents," Annals of Mathematics, vol. 174, no. 3, pp. 1571-1601, 2011.

[25] G. Tierno, "The paratingent space and a characterization of $C^{1}$ maps defined on arbitrary sets," Journal of Nonlinear and Convex Analysis, vol. 1, no. 2, pp. 129-154, 2000.

[26] S. Z. Shi, "Choquet theorem and nonsmooth analysis," Journal de Mathématiques Pures et Appliquées, vol. 67, no. 4, pp. 411-432, 1988.

[27] A. Balbás, M. Ballvé, and P. Jiménez Guerra, "Density theorems for ideal points in vector optimization," European Journal of Operational Research, vol. 133, no. 2, pp. 260-266, 2001.

[28] W. Rudin, Functional Analysis, McGraw-Hill, New York, NY, USA, 2nd edition, 1991.

[29] J. M. Borwein and A. S. Lewis, Convex Analysis and Nonlinear Optimization. Theory and Examples, Canadian Mathematical Society, Springer, New York, NY, USA, 2nd edition, 2006.

[30] D. T. Luc and P. H. Dien, "Differentiable selection of optimal solutions in parametric linear programming," Proceedings of the American Mathematical Society, vol. 125, no. 3, pp. 883-892, 1997. 


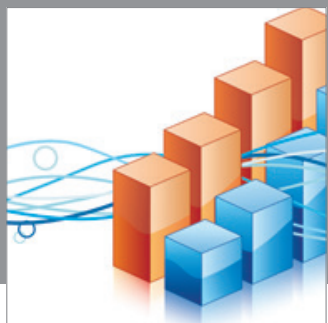

Advances in

Operations Research

mansans

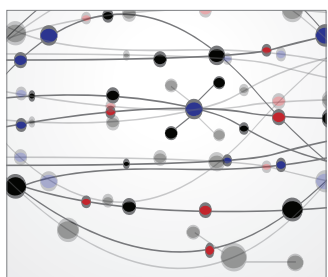

The Scientific World Journal
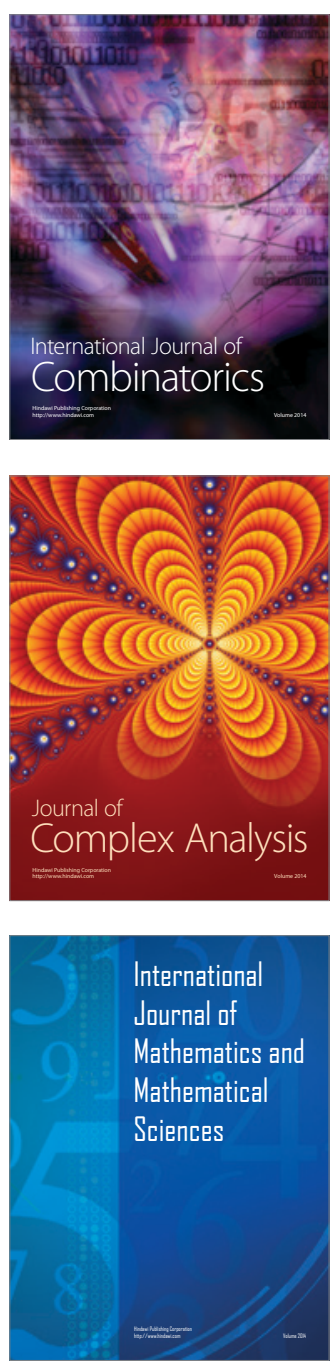
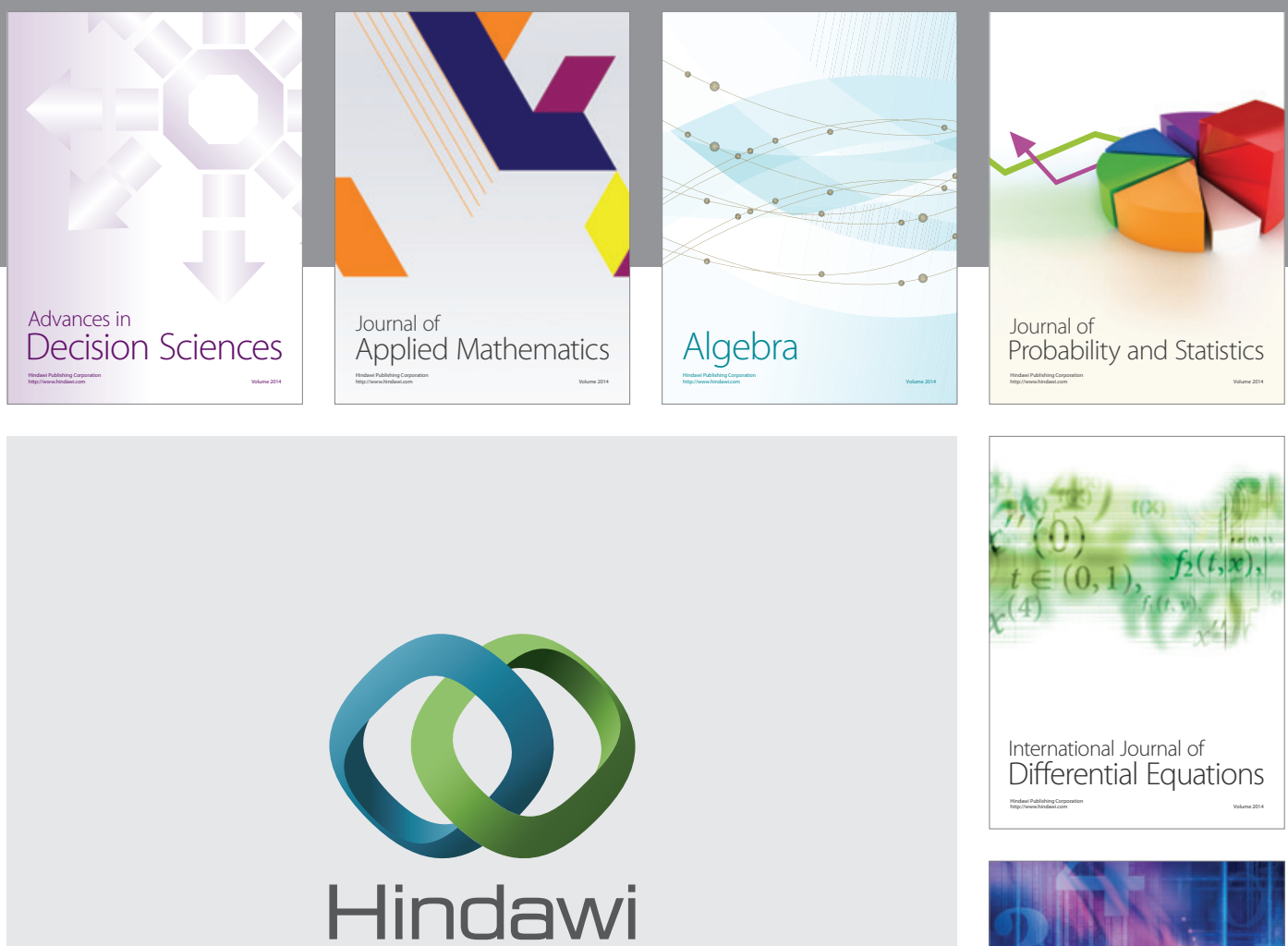

Submit your manuscripts at http://www.hindawi.com
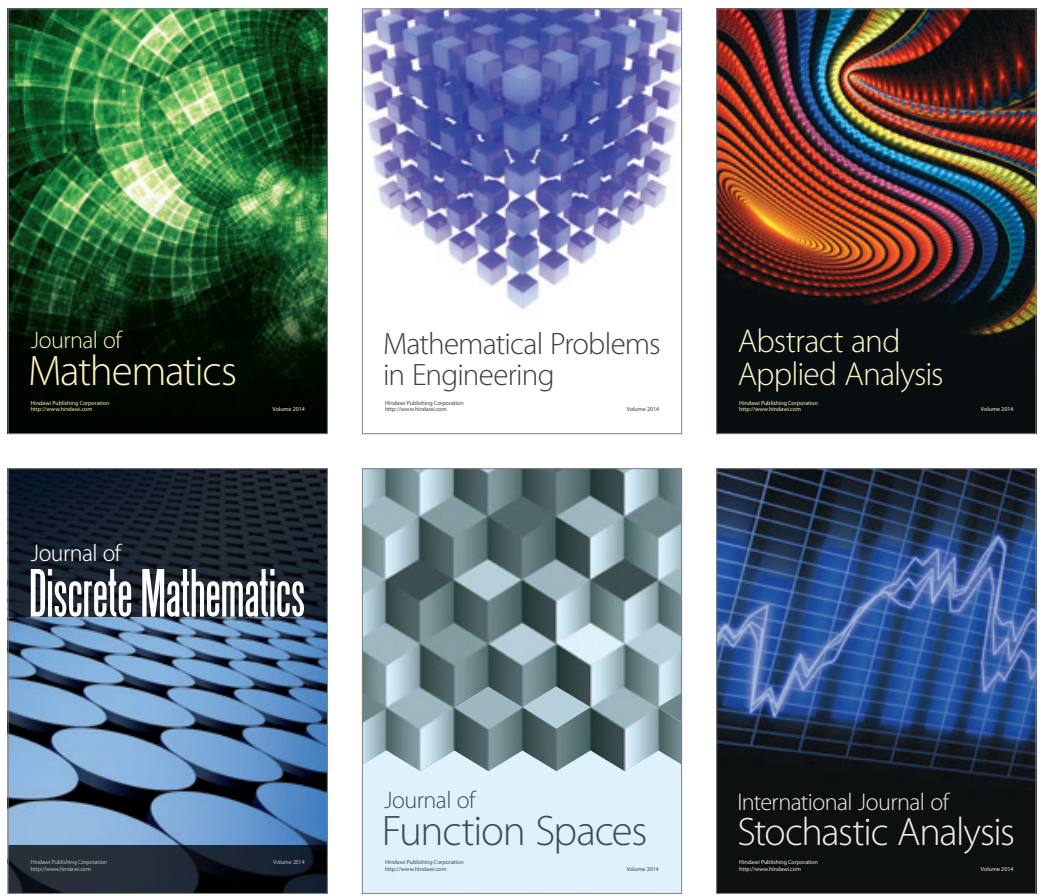

Journal of

Function Spaces

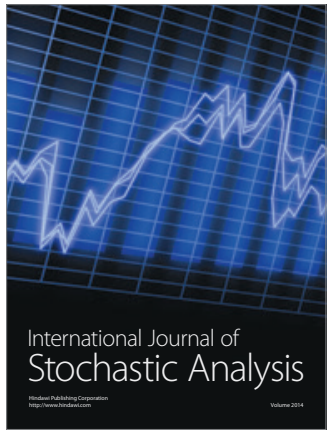

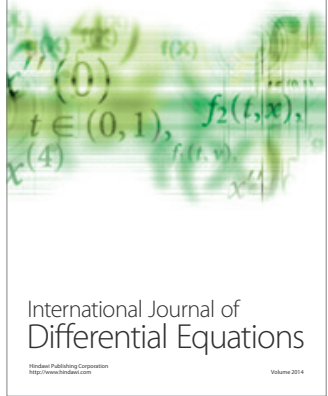
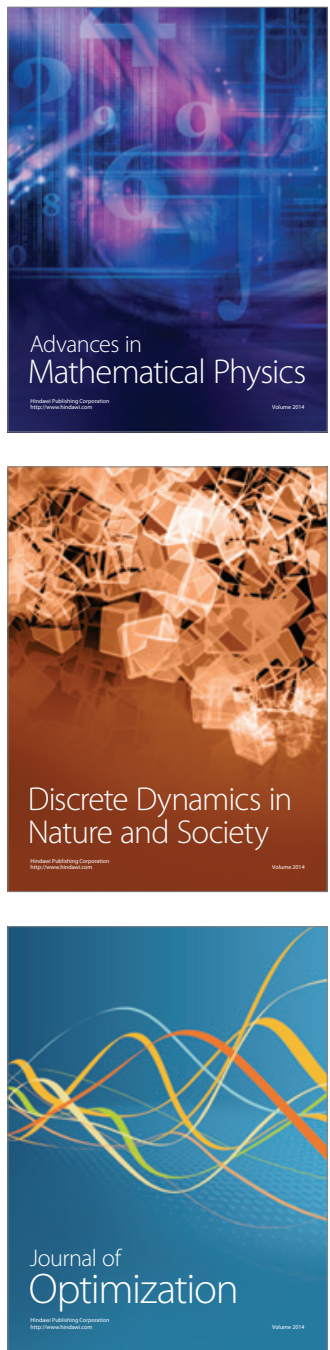